\title{
Complexity in high-temperature superconductors
}

\author{
G. Alvarez ${ }^{1}$, A. Moreo ${ }^{2}$, and E. Dagotto ${ }^{2}$ \\ ${ }^{1}$ Computer Science \& Mathematics Division, Oak Ridge National Laboratory \\ Oak Ridge, Tennessee 37831, USA \\ E-mail: alvarezcampg@ornl.gov \\ ${ }^{2}$ Condensed Matter Sciences Division, Oak Ridge National Laboratory, Oak Ridge, Tennessee 37831 and \\ Department of Physics and Astronomy, The University of Tennessee, Knoxville, Tennessee 37996, USA
}

Received August 25, 2005

\begin{abstract}
A spin-fermion and Landau-Ginzburg model for high-temperature superconductors are formulated and analyzed with unbiased numerical techniques. The study of the competition between $d$-wave superconductivity and antiferromagnetism in the clean limit suggests that the phase diagram is not universal. When chemical disorder is considered, a «clustered state» with short range order but without long range order appears leading to the possibility of a giant proximity effect. The influence of disorder on the spectral function is also addressed and results are compared to available angle resolved photoemission data for cuprates.
\end{abstract}

PACS: 74.20.De 74.20.Rp

Keywords: high- $T_{C}$ superconductivity, clustered states, pseudo-gap, giant effects.

\section{Introduction}

Understanding the physics of high-temperature superconductors (HTSs) has been one of the most important challenges in condensed-matter physics since the discovery of these materials in 1986 by J.G. Bednorz and K.A. Müller. Studies of other oxides have also shown very interesting and complex behavior [1]. In this paper it will be argued that inhomogeneities seem to play an important role in explaining many properties of these materials and that different inhomogeneous states could be stabilized in different $\mathrm{Cu}$-oxides, depending on coupling and quenched disorder strengths. Our computer simulations suggest that there is no unique way to transition from the antiferromagnetic (AF) to the superconducting regions of the phase diagram. There is also overwhelming experimental evidence for several unconventional regimes in HTSs, including a pseudo-gap region at temperatures above the superconducting phase, and a largely unexplored glassy state separating the parent antiferromagnet from the SC phase at low hole-doping $x$. Moreover, neutron scattering studies have revealed «stripes» of charge in Nd-LSCO [2-5], but scanning tunneling microscopy (STM) experiments $[6,7]$ indi- cate «patches» in Bi2212. All this is consistent with the results obtained here as will be explained below.

The arguments will be supported by the study of phenomenological models with unbiased numerical methods. Two models are used, one with itinerant fermions and the other without, and the conclusions are similar in both. The inclusion of chemical disorder leads to a ground state that presents short range but no long range order of any kind. The properties and importance of this state of the system will be discussed in detail.

In addition, recent investigations unveiled another remarkable property of HTSs that defies conventional wisdom: the existence of giant proximity effects (GPE) in some cuprates [8-10], where a supercurrent in Josephson junctions was found to run through non-SC Cu-oxide-based thick barriers. This contradicts the expected exponential suppression of supercurrents with barrier thickness beyond the short coherence length of $\mathrm{Cu}$-oxides. We will review a recent explanation [11] based on a description of the glassy state as containing SC areas. This nanoscale inhomogeneous state leads to colossal effects in cuprates, formally similar to colossal magnetoresistive manganites [12-14]. 
The dependence of $T_{C}$ with the number of Copper oxide layers will also be reviewed. In finite systems we have found that $T_{C}$ indeed increases rapidly with an increasing number of layers.

The form of the spectral functions in the presence of competing $\mathrm{AF}$ and $\mathrm{SC}$ states is presented following Ref. 15. One of the main results of this section is that the spectral function of the system without quenched disorder cannot reproduce the angle-resolved photoemission spectroscopy (ARPES) data but that quenched disorder is indeed crucial.

Studies of the $t-J$ model have revealed SC and striped states [16,17] evolving from the undoped limit. Then, it is reasonable to assume that $\mathrm{AF}, \mathrm{SC}$, and striped states are dominant in cuprates, and their competition regulates the HTS phenomenology. However, further computational progress using basic models is limited by cluster sizes that cannot handle the nanoscale structure unveiled by STM experiments. Considering these restrictions, here a phenomenological approach will be pursued to understand how these phases compete, incorporating the quenched disorder inevitably introduced by chemical doping. This effort unveils novel effects of experimental relevance, not captured with firstprinciples studies. Hopefully, the results and methods reviewed here will jump start a more detailed computational analysis of phenomenological models in the high- $T_{c}$ arena, since most basic first-principles approaches, including Hubbard and $t-J$ investigations, have basically reached their limits, particularly regarding lattice sizes that can be studied.

\section{Microscopic model}

The analysis starts with a phenomenological model of itinerant electrons (simulating carriers) on a square lattice, locally coupled to classical order parameters:

$$
\begin{aligned}
H_{F}= & -t \sum_{<\mathbf{i} \mathbf{j}}\left(c_{\mathbf{i} \sigma}^{\dagger} c_{\mathbf{j} \sigma}+\text { h.c. }\right)+2 \sum_{\mathbf{i}} J_{\mathbf{i}} S_{\mathbf{i}}^{z} s_{\mathbf{i}}^{z}- \\
& -\sum_{\mathbf{i} \sigma} \mu_{\mathbf{i}} n_{\mathbf{i} \sigma}+\frac{1}{D} \sum_{\mathbf{i}, \alpha} \frac{1}{V_{\mathbf{i}}}\left|\Delta_{\mathbf{i} \alpha}\right|^{2}- \\
& -\sum_{\mathbf{i}, \alpha}\left(\Delta_{\mathbf{i} \alpha} c_{\mathbf{i} \uparrow} c_{\mathbf{i}+\alpha \downarrow}+\text { h.c. }\right),
\end{aligned}
$$

where $c_{\mathbf{i} \sigma}$ are fermionic operators, $s_{\mathbf{i}}^{z}=\left(n_{\mathbf{i} \uparrow}-n_{\mathbf{i} \downarrow}\right) / 2$, $n_{\text {i } \sigma}$ is the number operator, $D$ is the lattice dimension, and $\Delta_{\mathrm{i} \alpha}=\left|\Delta_{\mathrm{i} \alpha}\right| \exp \left(i \varphi_{\mathrm{i}}^{\alpha}\right)$ are complex numbers for the SC order parameter defined at the links $(\mathbf{i}, \mathbf{i}+\alpha)(\alpha-$ unit vector along the $x$ or $y$ directions). At $J_{\mathbf{i}}=0$, $d$-wave SC is favored close to half-filling since the pairing term involves nearestneighbor sites, as in any standard mean-field approximation to SC. The spin degrees of freedom (d.o.f.) are assumed to be Ising spins (de- noted by $S_{\mathrm{i}}^{z}$ ). Studies with 0(3) d.o.f. were found to lead to qualitatively similar conclusions, but they are more CPU time consuming. At $\Delta_{\mathrm{i} \alpha}=0$, the model, originally introduced by Moreo and collaborators $[18,19]$, has an antiferromagnetic ground state at $n=1$ and stripes at $n<1$. The parameters of relevance are $J_{\mathbf{i}}, \mu_{\mathbf{i}}$, and $V_{\mathbf{i}}$ ( $t$ is the energy unit), that carry a site dependence to easily include quenched disorder which is inevitable in chemically doped compounds as the cuprates. For a fixed configuration of classical fields, $\left\{\Delta_{\mathbf{i} \alpha}\right\}$ and $\left\{S_{\mathbf{i}}^{z}\right\}$, the one-particle sector is Bogoliubov diagonalized using the transformation:

$$
\begin{aligned}
c_{\mathbf{i} \uparrow} & =\sum_{n=1}^{n=N}\left\{a_{n}(\mathbf{i}) \gamma_{n \uparrow}-b_{n+N}^{*}(\mathbf{i}) \gamma_{n \downarrow}^{\dagger}\right\}, \\
c_{\mathbf{i} \downarrow} & =\sum_{n=1}^{n=N}\left\{b_{n}(\mathbf{i}) \gamma_{n \downarrow}+a_{n+N}^{*}(\mathbf{i}) \gamma_{n \uparrow}^{\dagger}\right\} .
\end{aligned}
$$

$a_{n}(\mathbf{i})$ and $b_{n}(\mathbf{i})$ in (2) are complex numbers and are chosen so that a Hamiltonian that is diagonal in $\gamma_{n \sigma}$ emerges. In the limit $T \rightarrow 0$, the Bogoliubov-de Gennes equations are recovered minimizing the energy [20-22]. Then, a
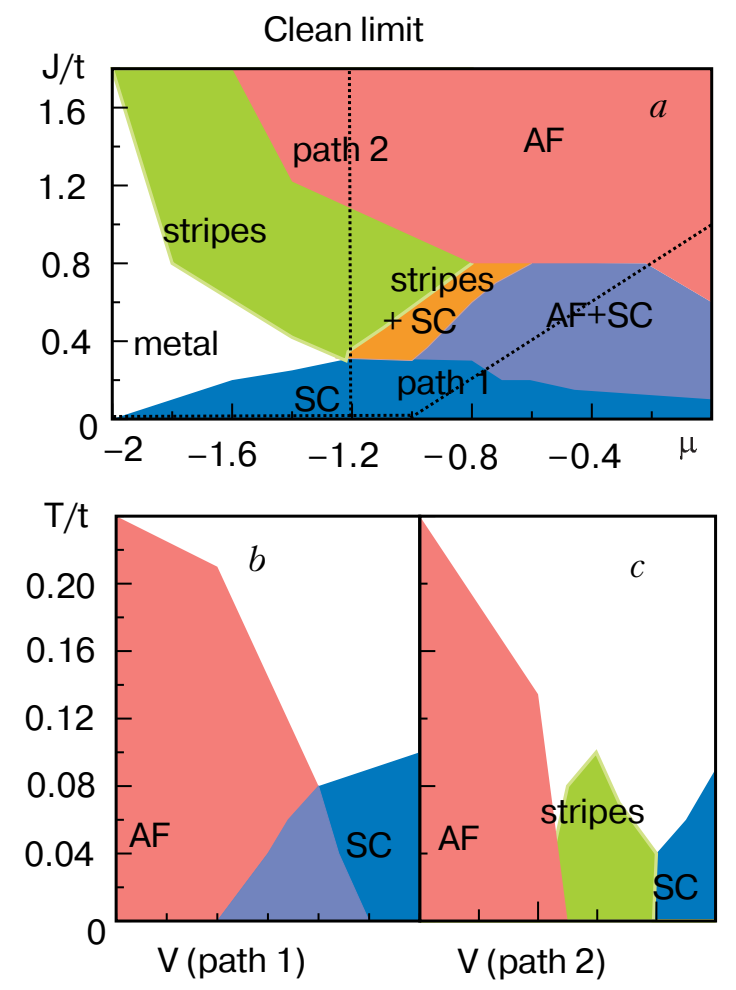

Fig. 1. MC phase diagram for Eq. (1) without disorder at low temperatures. Instead of presenting a three dimensional phase diagram we have chosen to present a two dimensional cut along $V=1-J / 2$ for simplicity. Five regions are observed: $\mathrm{AF}, d$-SC, stripes, coexisting $\mathrm{SC}+\mathrm{AF}$, coexisting stripes $+\mathrm{SC}$, and metallic $(a)$. MC phase diagram including temperature along «Path $1 »(b)$. MC phase diagram along «Path $2 »(c)$. Lattice sizes in all cases are $8 \times 8$ and $12 \times 12$. (From Ref. 11). 
standard Monte Carlo (MC) simulation similar to those for Kondo-lattice models is carried out (details in Ref. 12). One of the goals is to estimate $T_{c}$, as well as $T_{c}^{*}$, roughly defined as the temperature at which strong short-distance SC correlations develop.

\section{Clean limit}

Without quenched disorder, $V_{\mathbf{i}}, J_{\mathbf{i}}$ and $\mu_{\mathbf{i}}$ in Eq. (1) are site independent. The standard MC analysis carried out in these investigations reveals that in the clean limit the low temperature $(T)$ phase diagram, Fig. 1, $a$, has a robust AF phase for electronic densities $n \sim 1$ and a $d$-wave SC phase for $n<1$. The $d$-wave correlation function, defined as

$$
C_{s c}^{\alpha \beta}(\mathbf{m})=\sum_{\mathbf{i}}\left\langle\left|\Delta_{\mathbf{i}} \| \Delta_{\mathbf{i}+\mathbf{m}}\right| \cos \left(\varphi_{\mathbf{i}}^{\alpha}-\varphi_{\mathbf{i}+\mathbf{m}}^{\beta}\right)\right\rangle,
$$

was used to estimate $T_{c}$ as the temperature at which $\mathrm{d}$-wave correlations at the largest distances for the lattices considered here are $5 \%$ of their maximum value at $|\mathbf{m}|=0$. The $5 \%$ criterion is arbitrary but other criteria lead to identical qualitative trends, slightly shifting the phase diagrams. $T^{*}$ is deduced similarly, but using the shortest nonzero distance correlation function $(|\mathbf{m}|=1)$. The Néel temperature, $T_{N}$, associated with the classical spins was defined by the drastic reduction ( $\leq 5 \%$ of $|\mathbf{m}|=0$ value) of the long-distance spin order using:

$$
C_{S}(\mathbf{m})=\sum_{\mathbf{i}}\left\langle S_{\mathbf{i}}^{z} S_{\mathbf{i}+\mathbf{m}}^{z}\right\rangle,
$$

while $T_{N}^{*}$ relates to short-range spin order. The results presented in Fig. 1, $a$ are not surprising since these states are favored explicitly in Eq. (1) by the second and fifth terms, respectively. However, the phase diagram presents several nontrivial interesting regions: (i) Along «Path 1» in Fig. 1,a, the AF-SC transition occurs through local coexistence, with tetracritical behavior (Fig. 1,b) [23]. (ii) Along «Path 2» the $\mathrm{AF}-\mathrm{SC}$ interpolating regime has alternating doped and undoped stripes (stripes in MC data are deduced from spin and charge structure factors, and low- $T$ MC snapshots), and a complex phase diagram, Fig. 1,c. These stripes evolve continuously from the $V=0$ limit that was studied before by Moreo et al., and as a consequence we refer the readers to Ref. 18 for further details on how stripes were identified. It remains to be investigated if these stripes, involving $\mathrm{SC}$ and $\mathrm{AF}$ quasi-1D lines, are originated by the same mechanisms as those widely discussed before in the high- $T_{C}$ literature [17, 24-28]. At $V \neq 0$, the doped regions of the stripes have nonzero SC amplitude at the mean-field level [29]. In view of the dramatically different be- havior along Paths 1 and 2, we conclude that in our model there is no unique $A F \rightarrow S C$ path. This is in agreement with experiments since $\mathrm{La}_{2} \mathrm{Sr}_{x} \mathrm{CuO}_{4}$ (LSCO) and others have stripes, $[2-5,30]$ while $\mathrm{Ca}_{2-x} \mathrm{Na}_{x} \mathrm{CuO}_{2} \mathrm{Cl}_{2}$ has a more complex inhomogeneous pattern [7]. Both, however, become SC with increasing $x$. This suggests that the underdoped region of $\mathrm{Cu}$-oxides may not be universal.

\section{Disordered system}

Our results become even more interesting upon introducing quenched disorder. Disorder may have several forms, but here we mimic Sr-doping in singlelayers. $\mathrm{Sr}^{2+}$ replaces $\mathrm{La}^{3+}$, above the center of a $\mathrm{Cu}$-plaquette in the $\mathrm{Cu}-$ oxide square lattice. Then, as hole carriers are added, a hole-attractive plaquette-centered potential should also be incorporated as sketched in Fig. 2. Near the center of this potential, $n$ should be sufficiently reduced from 1 that, phenomenologically, tendencies to SC should be expected. To interpolate between the $\mathrm{SC}$ central plaquette and $\mathrm{Sr}$ the $\mathrm{AF}$ background, a plaquette «halo» with no dominant tendency was introduced. Parameters are chosen such that the blue (black) region favors superconductivity, $(J, V, \mu)=(0.1,1.0,-1.0)$, with a surrounding region where $(J, V, \mu)=(0.1,0.1,-0.5)$ with no order prevailing. The impurity is embedded in a background that favors the AF state, $(J, V, \mu)=(1.0,0.1,0.0)$. However, the overall conclusions found here are simple, and independent of the disorder details.

The phase diagram obtained with the Monte Carlo simulation is shown in Fig. 3, $a$. The similarity with the widely accepted phase diagram of the cuprates is clear. The disorder has opened a hole-density «window» where none of the two competing orders dominates. Inspecting «by eye» the dominant MC configurations (snapshots) at low- $T$ in this intermediate

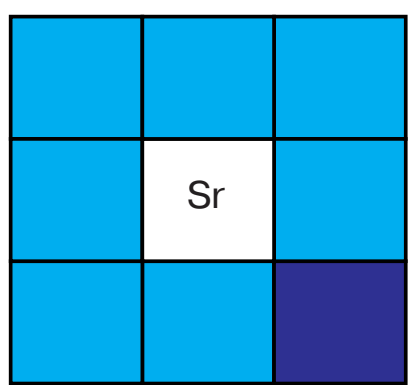

Fig. 2. Schematic representation of $\mathrm{Sr}$ doping. A chemical dopant (Sr) will not only disorder one site in the $\mathrm{CuO}_{2}$-plane, but also neighboring ones, motivating the introduction of «plaquette»-like disorder configurations that affect nearby sites (right/bottom square). 

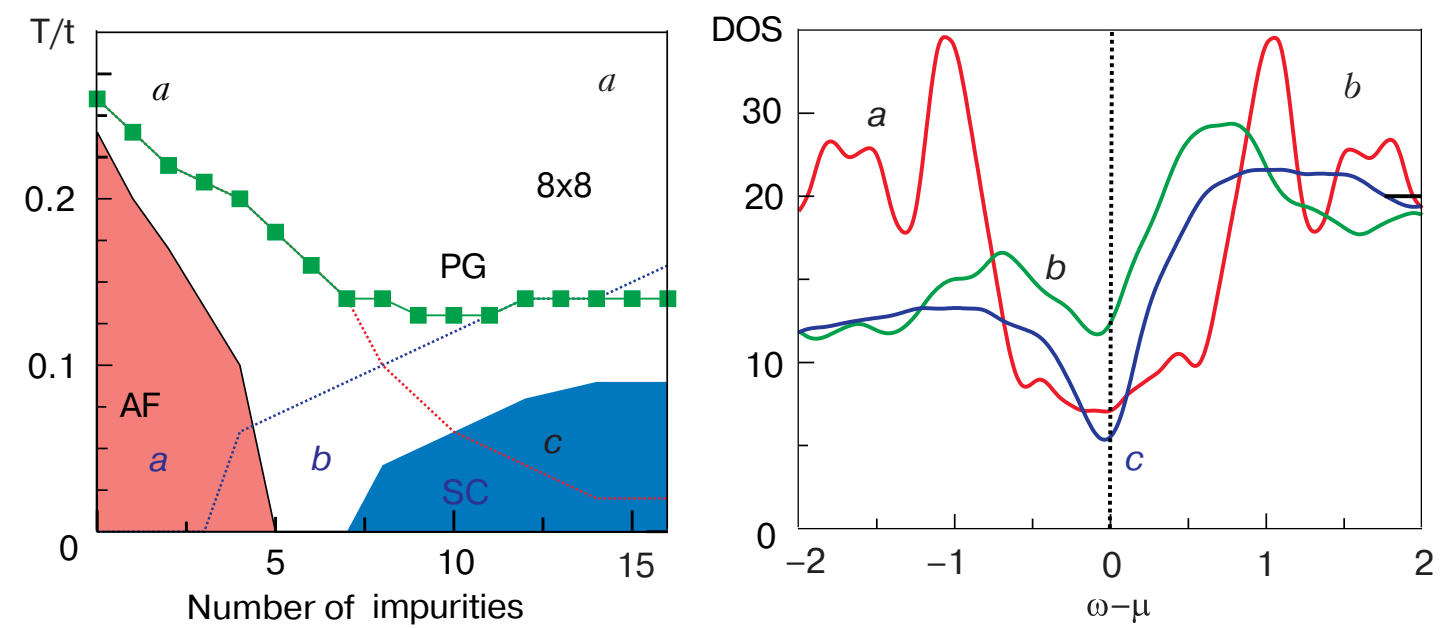

Fig. 3. MC phase diagram for model Eq. (1) including quenched disorder (lattices studies are $8 \times 8$ (results shown) and $12 \times 12$ ). Shown are $T_{C}$ and $T_{N}$ vs. number of impurities (equal to number of holes). The $\mathrm{SC}$ and $\mathrm{AF}$ regions with short-range order (dashed lines), and $T^{*}$ as obtained from the PG (dot-dashed line) are also indicated $(a)$. DOS at points $a, b$, and $c$ of $(a)$, with a PG (b); (from Ref. 11).

regime reveals a patchy system with slowly evolving islands of $\mathrm{SC}$ or $\mathrm{AF}$, and random orientations of the local order parameters, leading to an overall disordered «clustered» state. In Fig. 3, $a$, a new temperature scale $T^{*}$ at which the fermionic density-of-states (DOS) develops a pseudogap (PG) (Fig. 3,b) was also unveiled. The $\mathrm{AF}$ and $d$-SC regions both have a gap (smeared by $T$ and disorder, but nevertheless with recognizable features). But even the «disorder» regime (case $b$ in Fig. $3, b$ ) has a PG. MC snapshots explains this behavior: in the disordered state there are small SC or AF regions, as explained above. Locally each has a smeared-gap DOS, either AF or SC. Not surprisingly, the mixture presents a PG. The behavior of $T^{*}$ vs. $x$ is remarkably similar to that found experimentally. The cuprates'PG may arise from an overall-disordered clustered state with local AF or SC tendencies, without the need to invoke other exotic states. This PG is correlated with robust short-range correlations (dashed lines in Fig. 3, $a$, see caption for details). For a detailed study of the $T^{*}$. and the pseudo-gap formation in the clean limit, see Ref. 31.

\section{Landau-Ginzburg model}

The results reported thus far, based on Eq. (1), have already revealed interesting information, namely the possible paths from $\mathrm{AF}$ to $\mathrm{SC}$, and a proposed explanation of the glassy state as arising from the inevitable quenched disorder in the samples. However, the inhomogeneous nature of the clustered region suggests that percolative phenomena may be at work, and larger clusters are needed. To handle this issue, another model containing only classical d.o.f. is proposed, with low-powers interactions typical of Landau-Ginzburg (LG) approaches:

$$
\begin{gathered}
H=r_{1} \sum_{\mathbf{i}}\left|\Delta_{\mathbf{i}}\right|^{2}+\frac{u_{1}}{2} \sum_{\mathbf{i}}\left|\Delta_{\mathbf{i}}\right|^{4}+\sum_{\mathbf{i}, \alpha} \rho_{2}(\mathbf{i}, \alpha) S_{\mathbf{i}} S_{\mathbf{i}+\alpha}- \\
-\sum_{\mathbf{i}, \alpha} \rho_{1}(\mathbf{i}, \alpha)\left|\Delta_{\mathbf{i}}\right|\left|\Delta_{\mathbf{i}+\alpha}\right| \cos \left(\Psi_{\mathbf{i}}-\Psi_{\mathbf{i}+\alpha}\right)+r_{2} \sum_{\mathbf{i}}\left|S_{\mathbf{i}}\right|^{2}+ \\
+\frac{u_{2}}{2} \sum_{\mathbf{i}}\left|S_{\mathbf{i}}\right|^{4}+u_{12} \sum_{\mathbf{i}}\left|\Delta_{\mathbf{i}}\right|^{2}\left|S_{\mathbf{i}}\right|^{2}
\end{gathered}
$$

The fields $\Delta_{\mathbf{i}}=\left|\Delta_{\mathbf{i}}\right| \exp \left(i \varphi_{\mathbf{i}}\right)$ are complex numbers representing the SC order parameter. The classical spin at site $\mathbf{i}$ is

$S_{\mathbf{i}}=\left|S_{\mathbf{i}}\right|\left(\sin \left(\theta_{\mathbf{i}}\right) \cos \left(\varphi_{\mathbf{i}}\right), \sin \left(\theta_{\mathbf{i}}\right)\left(\sin \left(\varphi_{\mathbf{i}}\right), \cos \left(\theta_{\mathbf{i}}\right)\right)\right.$. $\rho_{1}(\mathbf{i}, \alpha)=1-\rho_{2}(\mathbf{i}, \alpha)$ is used as the analog of $V=$ $=1-J / 2$ of the previous model to reduce the multiparameter character of the investigation, allowing an $\mathrm{AF}-\mathrm{SC}$ interpolation changing just one parameter. $\alpha$ denotes the two directions $\hat{x}$ and $\hat{y}$ in $2 d$, and also $\hat{z}$ for multilayers. $\rho_{2}(i, \alpha)$ was chosen to be isotropic, i.e., $\alpha$-independent.

Clearly, the lowest-energy state for $\rho_{2}=0$ is a homogeneous SC state (if $\left.\rho_{1}(\mathbf{i}, \alpha)=\rho_{1}^{0}>0\right)$. When $\rho_{1}=0$ the lowest-energy state is AF (if $\rho_{2}(i, \alpha)=\rho_{2}^{0}>0$ ). In the clean limit, this model was already studied in the $\mathrm{SO}(5)$ context, where the reader is referred for further details. Our approach without disorder has similarities with $\mathrm{SO}(5)$ ideas [23], where the $\mathrm{AF} / \mathrm{SC}$ competition as the cause of the high- $T_{c}$ phase diagram was extensively discussed, although nowhere in our investigations we need to invoke a higher symmetry group. The relevance of tetracriticality in $\mathrm{La}_{2} \mathrm{CuO}_{4+\delta}$ has also been remarked by E. Demler et al. [32] and Y. Sidis 
[33]. In the present work, disorder is introduced by adding a randomly selected bimodal contribution, i.e. $\rho_{2}(\mathbf{i}, \alpha)=\rho_{2}^{0} \pm W$, where $W$ is the disorder strength ( $W=0$ is the clean limit). It is expected that other forms of disorder will lead to similar results.

\section{Phase diagram}

The phase diagram for model Eq. (5) was calculated for both «weak» $\left(u_{12}=0.7\right)$ and «strong» coupling $\left(u_{12}=2.0\right)$. The clean limit $(W=0)$ and disordered case $(W \neq 0)$ were both studied. In all cases we found qualitative similarities to the phase diagram for the microscopic model studied previously. One important difference is that the miscroscopic model never indicated a first-order transition whereas the Landau-Ginzburg model showed both coexistence at «weak» coupling and a first-order transtion (see Fig. 4) between the competing orders at «strong» coupling. Another difference between the two models is that Eq. (5) cannot lead to doped-undoped stripes, but the more general case Eq. (1) does. However, both models share a similar phenomenology, and Eq. (5) can be studied on larger lattices.

\section{Clustered states}

The formation of clustered states in high- $T_{C}$ superconductors could explain the important challenges in the physics of these materials, as will be shown. The technical details concerning the addition of chemical disorder where explained above. Here the Landau-Ginzburg model will be used since it allows to study larger lattices. First, Fig. 5, $a$ depicts the ground state of the system at low temperature. The different colors indicate the different phases of the supercon-

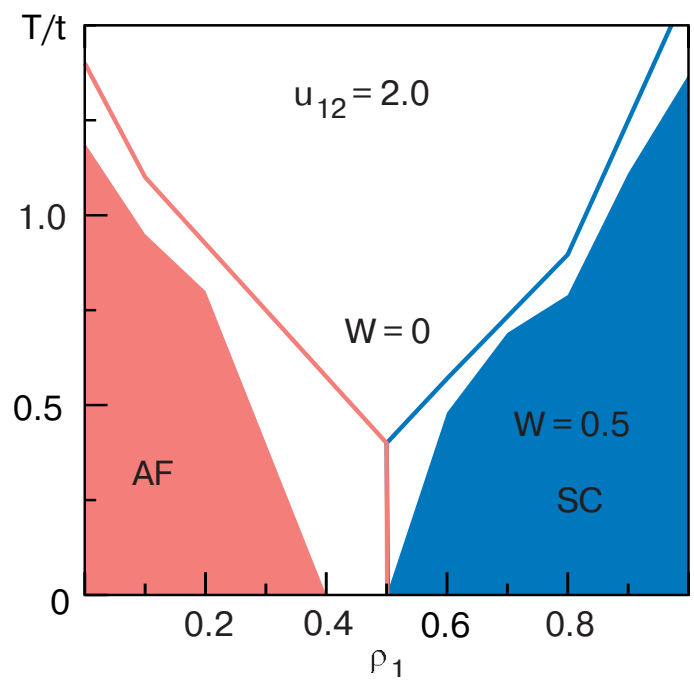

Fig. 4. MC phase diagram of model Eq. (5) at $u_{12}=2$. The clean case ( $W=0$, solid lines) is bicritical-like, but with disorder $W=0.5$ a clustered region between SC and AF opens as well (from Ref. 11).
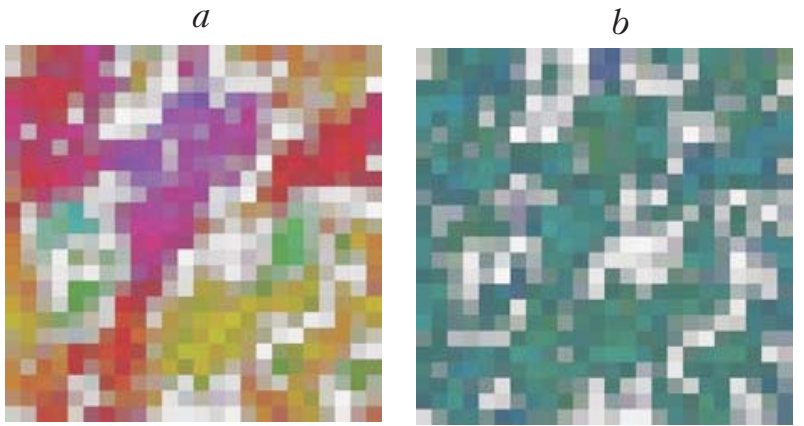

Fig. 5. MC snapshots are shown at $\Delta_{S C}^{\text {ext }}=0.0(a)$ and $\Delta_{S C}^{\text {ext }}=0.2(b)$, both at $T=0.1$ and $\rho_{2}=0.5$, using the same quencheddisorder configuration. Colors (or shades of gray) indicate the SC phase, while intensities are proportional to $\operatorname{Re}\left(\Delta_{\mathbf{i}}\right)$. The AF order parameter is not shown. The multiple-color nature of the left snapshot, reflects a SC phase randomly distributed (i.e. an overall non-SC state). However, a small external field rapidly aligns those phases, leading to a coherent state as shown on the right.

ducting order parameter, whereas the intensity indicates the amplitude of the order parameter. For simplicity the antiferromagnetic order parameter is not shown. It can be clearly seen that the system tends to form «clusters» even when the disorder is added randomly. The resulting state presents short range superconducting order but the system is not globally superconducting.

\section{Giant proximity effect in cuprates}

These «clustered» states could explain the giant proximity effect observed in some cuprates [8]. Consider a typical clustered state (Fig. 5, $a$ ) found by MC simulations in the disordered region. This state has preformed local SC correlations - nanoscale regions having robust SC amplitudes within each region, but no SC phase coherence between different regions rendering the state globally non-SC (the averaged correlation at the largest distances available, $C_{S C}^{\max }$, is nearly vanishing). Let us now introduce an artificial SC «external field», which can be imagined as caused by the proximity of a layer with robust SC order (e.g., comprised of a higher- $T_{C}$ material). In practice, this is achieved in the calculations by introducing a term $\left|\Delta_{S C}^{\text {ext }}\right| \sum_{\mathbf{i}} \rho_{1}(\mathbf{i}, \hat{z})\left|\Delta_{\mathbf{i}}\right| \cos \left(\psi_{\mathbf{i}}\right) \mid$, where $\Delta_{S C}^{\text {ext }}$ acts as an external field for SC. This field aligns the phases of the superconducting order parameter (Fig. 5,b) and the dependence of $C_{S C}^{\max }$ with $\Delta_{S C}^{\text {ext }}$ is simply remarkable (Fig. 6, $a$ ). While at points $e$ and $f$, located far from the SC region in Fig. 6 right, the dependence is the expected one for a featureless state (linear), the behavior closer to SC and small temperatures is highly nonlinear and unexpected. For example, at point $a$, 

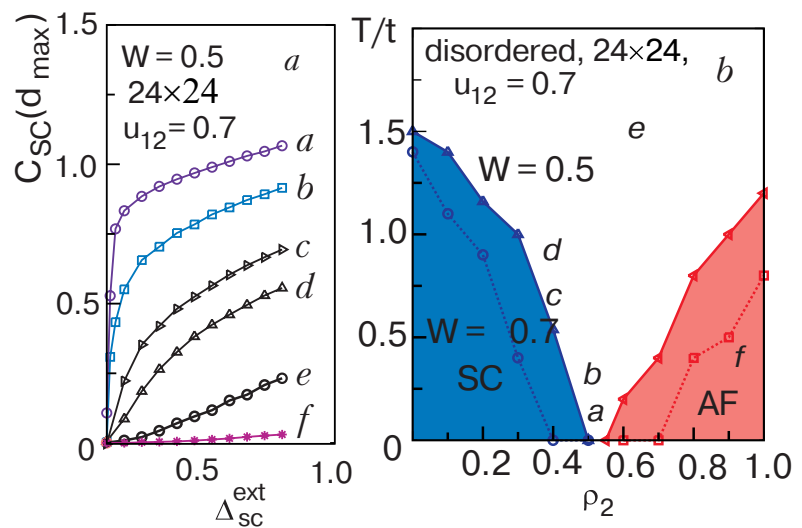

Fig. 6. $C_{S C}^{\max }$ vs. $\triangle \triangle_{S C}^{\text {ext }}$ (see text) on a $24 \times 24$ lattice, with $u_{12}=0.7$ and $W=0.5$, at the five points $a-f$ indicated on the right panel. A «colossal» effect is observed in $a$ and $b$ where the $\Delta_{S C}^{\text {ext }}=0$ state is «clustered». A much milder (linear) effect occurs far from the SC phase ( $e$ and $f)(a)$. The points in the phase diagram where $C_{S C}^{\max }$ was plotted (b). (from Ref. 11).

$C_{S C}^{\max }$ vs. $\Delta_{S C}^{\text {ext }}$ has a slope (at $\Delta_{S C}^{\text {ext }}=0.02$ ) which is $\sim 250$ times larger than at $e(\sim 13$ times larger than at $W=0$, same $T, \rho_{2}$, and $u_{12}$.).

The reason for this anomalous behavior is the clustered nature of the states. This is shown in the state Fig. 5, $b$, contrasted with $(a)$, where a relatively small field - in the natural units of the model - nevertheless led to a quick alignment of SC phases, producing a globally SC state, as can be inferred from the uniform color of the picture. Having preformed SC puddles vastly increases the SC susceptibility. Since Fig. 6 left was obtained in a trilayer geometry it is tempting to speculate that the proximity of SC layers to a non-SC but clustered state, can naturally lead to a GPE over long distances, as observed experimentally in a similar geometry [8-10].

\section{Multilayers}

The nanoscale clusters also lead to a proposal for explaining the rapid increase of $T_{c}$ with the number of $\mathrm{Cu}$-oxide layers $N_{\ell}$, found experimentally, at least up to 3 layers. In this effort, the MC phase diagrams of single-, bi-, and tri-layer systems described by Eq. (5), with and without disorder, were calculated using exactly the same parameters (besides a coupling $\rho_{2}(\mathbf{i}, \hat{z})$, equal to those along $\hat{x}$ and $\hat{y}$, to connect the layers). It was clearly observed that the single layer has a substantially lower $T_{c}$ than the bilayer. This can be understood in part from the obvious critical fluctuations that are stronger in $2 \mathrm{D}$ than $3 \mathrm{D}$. But even more important, cluster percolation at $W \neq 0$ is more difficult in $2 \mathrm{D}$ than $3 \mathrm{D}$ (since otherwise 2D disconnected

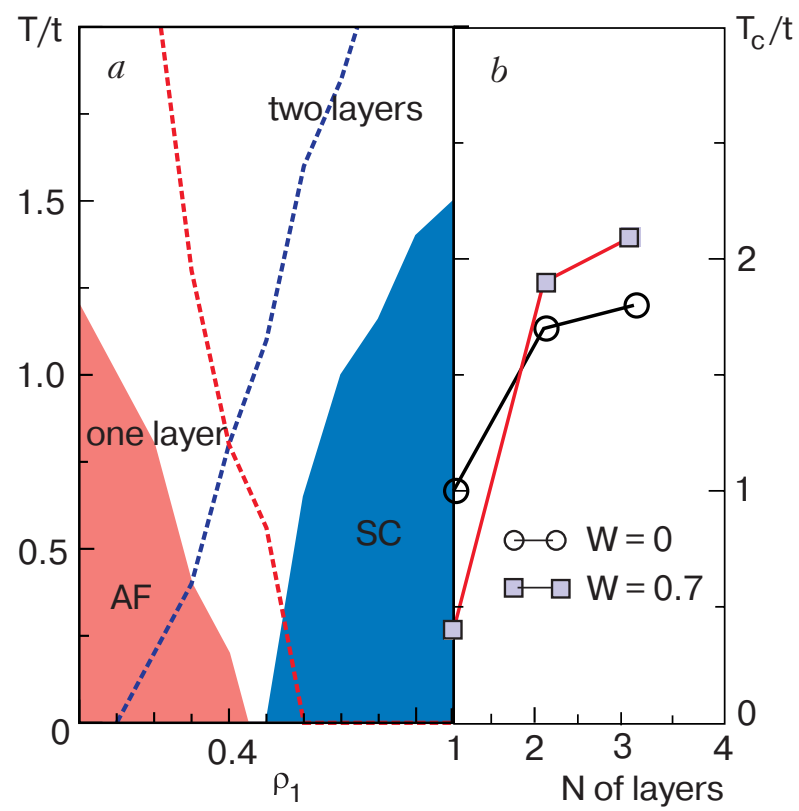

Fig. 7. MC phase diagram (for Eq.(5)) at $u_{12}=0.7$. Parameters are $r_{1}=-1, r_{2}=-0.85, u_{1}=u_{2}=1, W=0.5$ with one layer (solid colors) and two layers (dashed line). The addition of an extra layer increases the critical temperature of the superconductor as well as the Néel temperature $(a) . T_{c}$ vs. $N_{\ell}$ for $u_{12}=0.7, \rho_{2}=0.3$, and $24^{2} \times N_{l}$ clusters. Shown are results with $(\mathrm{W}=0.7)$ and without $(W=0.0)$ disorder $(b)$.

clusters may become linked through an interpolating cluster in the adjacent layer).

Then, in the phenomenological approach presented here it is natural that $T_{c}$ increases fast with $N_{\ell}$, when changing from 1 to 2 layers as shown in Fig. 7, $a$. This concept is even quantitative - up to a scale - considering the similar shape of $T_{c}$ vs. $N_{\ell}$ found both in the MC simulation (see Fig. 7,b) and in experiments [34]. Our $M C$ results suggest that the large variations of $T_{C} s$ known to occur in single-layer cuprates can be attributed to the sensitivity of $2 D$ systems to disorder. As $N_{\ell}$ increases (more layers are added), the influence of disorder decreases, both in experiments [35] and simulations.

\section{Spectral function for clustered states}

In this section, we will present the analysis of the one-particle spectral function, $A(\mathbf{k}, \omega)$, for several regimes of the phase diagram of Eq. (1). The discussion here follows closely Ref. 15. For general doping and interaction values this can only be done with the MC procedure described in the Section about the microscopic model. $A(\mathbf{r}, t)$ is defined by the expression:

$$
A(\mathbf{r}, t)=\left\langle\sum_{\mathbf{i}} c_{\mathbf{i} \sigma}^{\dagger}(t) c_{\mathbf{i}+\mathbf{r}, \boldsymbol{\sigma}}+\text { h.c. }\right\rangle .
$$


Applying the modified BdG transformation, Eq. (2), Eq. (6) is calculated using:

$$
A(\mathbf{r}, \omega)=\sum_{n} X_{n}(\mathbf{r}) \delta\left(\omega-E_{n}^{\uparrow}\right)+Y_{n}(\mathbf{r}) \delta\left(\omega+E_{n}^{\uparrow}\right),
$$

where

$$
X_{n}(\mathbf{r})=\sum_{\mathbf{i}} a_{n}^{*}(\mathbf{I}) a_{n}(\mathbf{I}+\mathbf{r}),
$$

and a similar expression is valid for $Y_{n}$. The eigenvalues of the «spin-up» one-electron sector are denoted by $E_{n}^{\uparrow}$. Eq. (7) can be Fourier-transformed to obtain $A(\mathbf{k}, \omega)$, but it is faster to do that after taking the average, and that route has been followed in the present work.

\section{Clean limit}

The phase diagram of Eq. (1) for the clean case (i.e. without quenched disorder) was presented in Fig. 1. The figure shows two «paths», which describe the transition from the $\mathrm{AF}$ to the $\mathrm{SC}$ phase. The first one crosses a region of longrange order with local $\mathrm{AF} / \mathrm{SC}$ coexistence, whereas the second one involves an intermediate «stripe» state [18]. We do not discuss here the exact nature of the stripe state, which may be horizontal or diagonal, depending on parameters such as doping and lattice size. For our purposes it is sufficient that an inhomogeneous state - stripe, phase separation or charge order - exists, and what its effects are with regards to experimental probes. Four representative points along those two paths (see Fig.1) were chosen and the corresponding spectral functions calculated.

Figure $8, a$ shows $A(\mathbf{k}, \omega)$ for the purely SC case $(J=0)$ for $\mu=-1$, leading to a uniform density $\langle n\rangle \approx 0.7$. Fig. $8, b$ is for the case when the system presents local $\mathrm{AF} / \mathrm{SC}$ coexistence (namely, both order

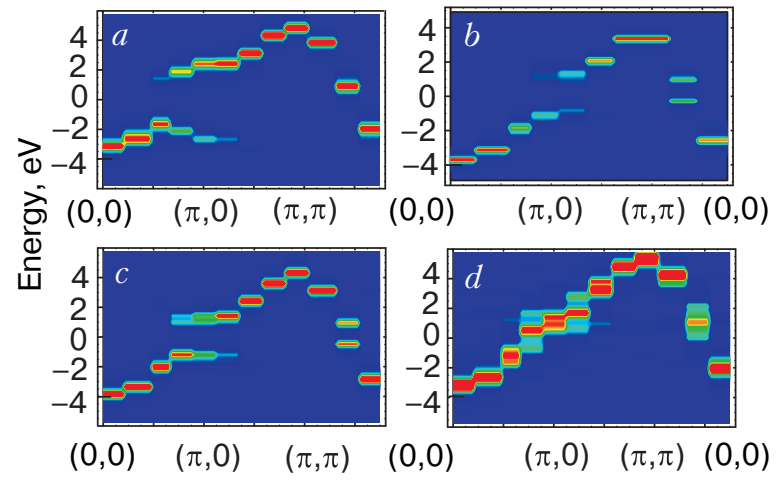

Fig. 8. $A(\mathbf{k}, 0)$, evaluated via MC, on an $8 \times 8$ lattice for $(a)$ $(J, V, \mu)=(0,1,-1)$ (SC state); $(J, V, m)=(0.6,0.7,-0.4)$, coexisting $\mathrm{AF} / \mathrm{SC}$ state $(b) ;(J, V, \mu)=(0.7,0.65,-0.3)$ $(\mathrm{AF})(c)$, and $(d)(J, V, \mu)=(1,0.5,-1.2)$, striped state (from Ref. 15). parameters simultaneously nonzero at the same site) and Fig. 8,c for the pure AF phase. The light color on the spots indicates large spectral weight, whereas the dark one indicates very low intensity. In Fig. 8,c, the AF gap can be clearly identified, together with the typical dispersion of the AF (upper branch), $E_{\mathbf{k}}= \pm \sqrt{\varepsilon_{\mathbf{k}}^{2}+J_{2}}$, which makes $E_{\mathbf{k}}$ gapped everywhere. This is in stark contrast to Fig. 8, $a$, where there are electronic states with appreciable intensity near the Fermi energy $\left(E_{F}\right)$ close to $(\pi / 2, \pi / 2)$, allowed by the symmetry of the pairing state. The «intermediate» state with local $\mathrm{AF} / \mathrm{SC}$ coexistence is not drastically different from the one with AF correlations only, and its resulting energy dispersion can be simply described by $E_{\mathbf{k}}= \pm \sqrt{\left(e_{\mathbf{k}}-\mu\right)^{2}+J^{2}+\Delta_{\mathbf{k}}^{2}}$ once $\Delta_{\mathbf{k}}$ is known. This conclusion is not supposed to change using the SO(3)-symmetric spin model.

Similarly, along path 2 of Fig. 1, $a$ point in the phase diagram with striped order was chosen, and the corresponding spectral density is given in Fig. 8, $d$. This result compares very well with previous calculations, (Ref. 19, Fig. 7): for instance, the system presents a Fermi surface crossing near $(\pi, 0)$. Whereas the results from Figs. 8, $a, c$ refer to generally well-understood phases of the cuprate phase diagram, Figs. 8, $b, d$ are of relevance for the discussion related to the intermediate state, since they are both candidates for the intriguing phase in between.

For comparison, ARPES data from Ref. 36 for $\mathrm{La}_{2-x} \mathrm{Sr}_{x} \mathrm{CuO}_{4}$ are reproduced in Fig. 9. For very low doping $x=0.03$ (just inside the spin-glass insulating (SGI) phase) a flat band is observed close to $-0.2 \mathrm{eV}$ in addition to a lower branch (energy $\sim-0.55 \mathrm{eV}$ ), which is already present in the $x=0$ limit and therefore can be safely identified with the lower Hubbard band. As $x$ is increased even further, the lower branch

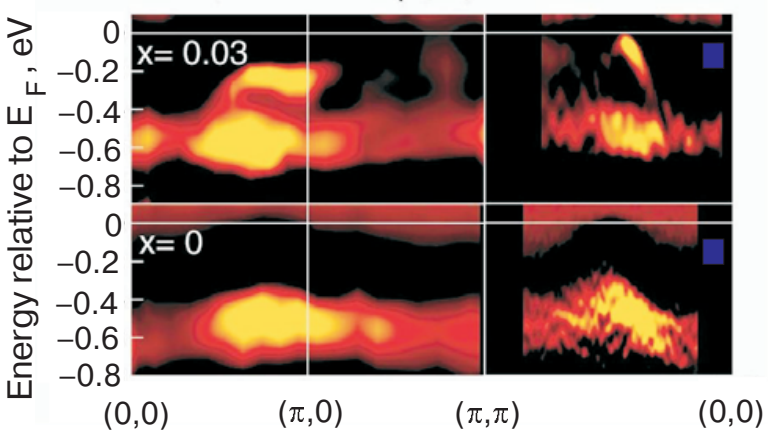

Fig. 9. Experimental ARPES spectra for LSCO with $x=0$ and $x=0.03$. Note the development of a (flat) second highintensity branch near $(\pi, 0)$ and the emergence of a strongly dispersive signal at the Fermi level as the system is doped away from the half-filled insulator (reproduced from Ref. 36). 
retains its energy position, but gradually loses its intensity until it is almost completely invisible after the onset of the SC phase at $x=0.06$ [37]. In contrast, the second branch gains in intensity with doping, and also moves continuously closer toward the Fermi level; at the same time it starts to develop a coherence peak, which is clearly visible for optimal doping. The main experimental result here, namely the existence of two branches near $(\pi, 0)$, cannot be reproduced using spatially homogeneous models as demonstrated above. The cases of $\mathrm{AF}, \mathrm{SC}$ and coexisting $\mathrm{AF}+\mathrm{SC}$ states all show only one branch below $E_{F}$ nearby $(\pi, 0)$. This was already seen in Figs. 8, $a-8, c$ for the MC data and is seen again in the exact dispersion for those ordered phases [11].

If stripe configurations are considered, as in Fig. 8,d (MC data), there will appear two branches near $E_{F}$, but the form of the dispersion is clearly different from the experimental data in Fig. 9. The same occurs if instead of using data from a Monte Carlo simulation, a perfect stripe configuration is studied as in Ref. 11. The investigation of $A(\mathbf{k}, \omega)$ for a spin-fermion model, related to Eq. (1), with $\Delta_{i, \alpha}=0$ but retaining the $\mathrm{SO}$ (3) spin symmetry, has been done carefully in Ref. 19. Again, stripe phases were found for certain parameters and while in some cases the existence of two branches near $(\pi, 0)$ was reported, certainly there are no indications of «nodal» quasiparticles at $(\pi / 2, \pi / 2)$. Then, stripes alone are not an answer to interpret the results of Yoshida et al. As a consequence, we conclude that neither local $\mathrm{AF}+\mathrm{SC}$ coexistence nor stripes can fully account for the ARPES results in the low-doping limit and alternative explanations should be considered.

Beyond the results already described, ARPES also provides surprising insights/results for momenta other than $(\pi, 0)$ (Fig. 9). Along the Brillouin zone diagonal, a dispersive band crossing $E_{F}$ is found already in the SGI phase. The FS-like feature consists of a small arc centered at $\sim(\pi / 2, \pi / 2)$; surprisingly, as more holes are added, this arc does not expand, but simply gains spectral weight. This increase in spectral intensity is roughly proportional to the amount of hole-doping for $x \leq 0.1$, although it grows more strongly thereafter. This observed increase in spectral weight is in relatively good agreement with the hole concentration $n_{H}$ derived from Hall measurements and was interpreted as a confirmation of the hole transport picture. Below, however, we will provide a different explanation for this behavior.

The aforementioned large gap $(\Delta \approx 0.2 \mathrm{eV})$ at $(\pi, 0)$, together with the existence of the apparent gapless excitations around $(\pi / 2, \pi / 2)$ is the essence of the PG problem. The shrinking of this gap and the concomitant appearance of a coherence peak has, for example, been interpreted as the evolution of a strongly coupled SC (at low doping) into a conventional BCS-SC at optimal doping. In this scenario, the large gap size directly reflects a large pairing scale, whereas the smallness of $T_{C}$ is attributed to the preponderance of phase-fluctuations in such a regime, which would rule out the existence of a phase-coherent SC condensate at higher temperatures. Alternatively, this gap may be regarded as the signal of a hidden order, which is not otherwise manifested. In other words, the relatively large excitation gap $\Delta_{P G}$ is explained in terms of (i) a large SC gap $\Delta_{P G}=\Delta_{S C}$ itself, or (ii) $\Delta_{P G}=\Delta_{S C}+\Delta_{h o}$, with a large, $x$-dependent hidden order gap $\Delta_{h o}$ whereas (iii) a mixed-state scenario, strongly influenced by disorder, leaves open the possibility that it is the (local) chemical potential that determines the PG physics.

\section{Disordered System}

Since calculations for $A(\mathbf{k}, \omega)$ in the clean limit do not agree with ARPES measurements, we turn our attention to a system with quenched disorder. The impact of quenched disorder is realized by tuning the coupling constants $J_{i}$ and $V_{i}$ in Eq.(1), as explained before.

When disorder is added, (the reader should consider again Fig. 3,a) a region between the $\mathrm{SC}$ and $\mathrm{AF}$ phases opens, where none of the competing order dominates and both regimes coexist in a spatially separated, mixed-phase state. This «glassy» state was discussed in detail in a previous section above, where it was suggested that it leads to «colossal effects». The pronounced susceptibility of such mixed-phase states towards applied «small» perturbations is wellknown and is, e.g., often regarded as the driving force behind

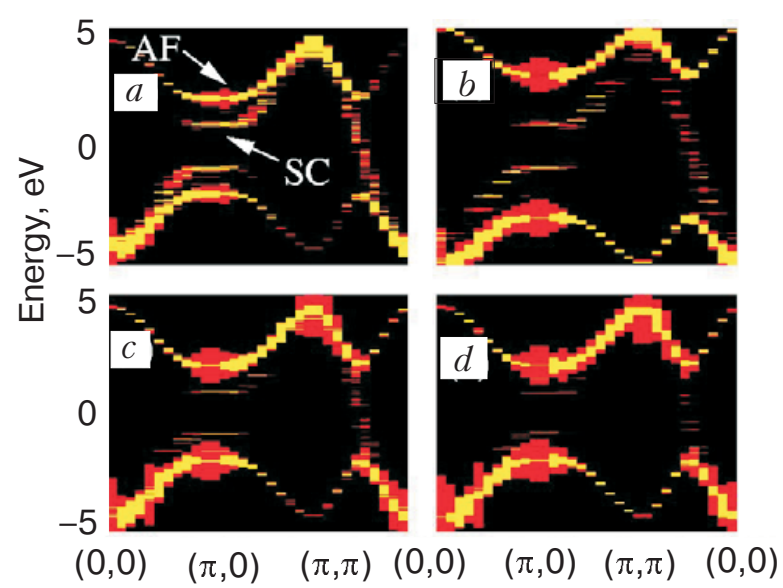

Fig 10. Distribution of $A(\mathbf{k}, 0)$ for a single configuration of classical fields, corresponding to a SC region of size $12 \times 12$ $(a), 8 \times 8(b), 7 \times 7(c)$ or $5 \times 5(d)$ on a $22 \times 22$ lattice (i.e., $30 \%, 15 \%, 10 \%$ or $5 \% \mathrm{SC}$, respectively). Shown is $E$ vs. $\mathbf{k}$ along $(0,0) \rightarrow(\pi, 0) \rightarrow(\pi, \pi) \rightarrow(0,0)$ (from Ref. 15). 
«colossal magneto-resistance» in manganites [13]. To simplify the study and be able to access larger systems, we will consider a single SC cluster embedded in an $\mathrm{AF}$ background and also consider a fixed or «frozen» configuration of the classical fields (both AF and $\mathrm{SC}$ ). When a $12 \times 12 \mathrm{SC}$ region is placed on an $\mathrm{AF}$ background (total lattice size is $22 \times 22$ ), the resulting distribution of $A(\mathbf{k}, \omega)$ is as shown in Fig. 10,a. The contribution from the AF background is clearly distinguishable from that of the SC island, since it is present even when the $\mathrm{SC}$ region is removed. The $\mathrm{SC}$ cluster induces a second «flat band» - quite typical for gapped systems - near $E_{F}$, along the $(0,0) \rightarrow(\pi, 0)$ direction. That this flat band is indeed produced by the SC island is verified by decreasing the size of the island to $8 \times 8$ (Fig. 10,b), $7 \times 7$ (Fig. 10,c) and finally for $5 \times 5$ (Fig. 10,d), upon which this signal gradually decreases (the cases $9 \times 9$ and $11 \times 11$ give very similar results to $12 \times 12$ and are not shown.). The spectral intensity related to the surrounding $\mathrm{AF}$ «bath» concurrently decreases, in agreement with experimental observations [37].

Therefore, even the simplest possible mixed-phase state can qualitatively account for the observed ARPES data. It is also interesting to note that SC signals comparably in strength with the ones stemming from the AF band, are only found for rather large SC blocks, encompassing at least $20 \%$ space of the whole system. From this point of view, even in the strongly underdoped limit at $x=0.03$, the relative amount of the SC phase has to be quite substantial already.

\section{Conclusions}

Simple phenomenological models for phase competition showed that - depending on details - different cuprates could have stripes, local coexistence, first-order transitions, or a glassy clustered state interpolating between $\mathrm{AF}$ and SC phases (Fig. 11,a). This clustered state in its simplest form is composed of superconducting areas on an antiferromagnetic background as shown in Fig. 11,b, and seems crucial to understand many aspects of high-temperature superconductors. For example, clustered states, which form below a temperature $T^{*}$, give rise to a pseudo-gap in the density of states. The study also revealed the possibility of giant proximity effects in materials where the SC state competes with another ordered state (here the AF state). This could be observed in structures fabricated with SC and non-SC (underdoped) films in close proximity, such that the bulk superconductivity in the SC film acts as the «external field» to orient the SC phases in the clustered state. Furthermore, the results provided a simple explanation for the increase of $T_{c}$ with the number of layers.
$\mathrm{T} / \mathrm{t}$

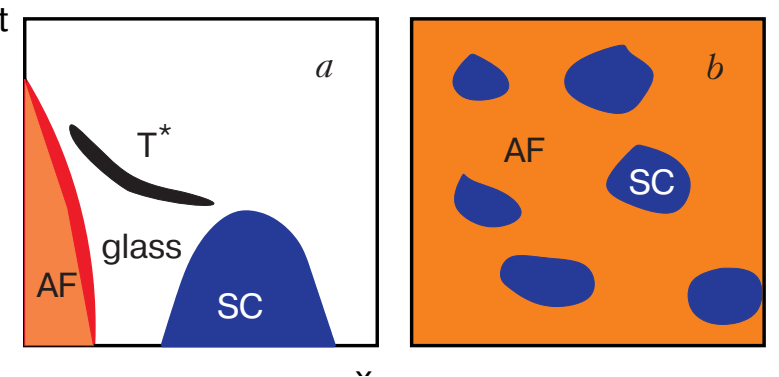

Fig. 11. Schematic representation of the phase diagrams that our models show in the dirty limit. Of particular interest is the glassy region, proposed to be a mixture of $\mathrm{SC}$ and $\mathrm{AF}$ clusters, and the $T^{*}$ where local order starts upon cooling. This phase diagram has formal similarities with those proposed before for manganites with antiferromagnetism vs. Ferromagnetism competition [12,13], and certainly it is in excellent agreement with the experimental phase diagram of $\operatorname{LSCO}(a)$. Schematic representation of the «glassy» state that separates the SC and AF regions. The SC clusters (dark regions) usually have different phases $(b)$.

The spectral function in the clustered phase with superconducting clusters and an antiferromagnetic background presented two components in the data for different concentrations of carriers. This result is compatible with photoemission experiments for $x=0.03$ LSCO, that reveal spectral weight in the node direction of the $d$-wave superconductor even in the insulating glassy regime [36]. Nodal $d$-wave SC particles surviving to low $x$ was observed in Ref. 38 .

It has already been found that clustered states are crucial in manganites and other compounds [39], and this analysis predicts its potential relevance in HTSs as well. The theoretical and experimental investigations of transition metal oxides in recent years point towards self-organized phenomena that usually manifest in the form of inhomogeneous states, revealing the intrinsic complexity of cuprates and manganites, and likely several other transition metal oxides.

The Oak Ridge National Laboratory is managed by UT-Battelle, LLC, for the U.S. Department of Energy under Contract DE-AC05-00OR22725. A.M. and E.D. are supported by NSF grant DMR-0312333.

\section{E. Dagotto, Science 309, 260 (2005).}

2. J.M. Tranquada, B.J. Sternlieb, J.D. Axe, Y. Nakamura, and S. Uchida, Nature 375, 561 (1995).

3. V. Emery, S.A. Kivelson, and H.Q. Lin, Phys. Rev. Lett. 64, 475 (1990).

4. S.A. Kivelson, I.P. Bindloss, E. Fradkin, V. Oganesyan, J.M. Tranquada, A. Kapitulnik, and C. Howald, Rev. Mod. Phys. 75, 1201 (2003).

5. S.A. Kivelson, E. Fradkin, and V.J. Emery, Nature 393, 550 (1998). 
6. K. Lang, V. Madhavan, J.E. Hoffman, E.W. Hudson, H. Eisaki, S. Uchida, and J.C. Davis, Nature 415, 412 (2002).

7. Y. Kohsaka, K. Iwaya, S. Satow, T. Hanaguri, M. Azuma, M. Takano, and H. Takagi, Phys. Rev. Lett. 93, 097004 (2004).

8. I. Bozovic, G. Logvenov, M.A.J. Verhoeven, P. Caputo, E. Goldobin, and M. Beasley, Phys. Rev. Lett. 93, 157002 (2004).

9. R.S. Decca, H.D. Drew, E. Osquiguil, B. Maiorov, and J. Guimpel, Phys. Rev. Lett. 85, 3708 (2000).

10. J. Quintanilla, K. Capelle, and L. Oliveira, Phys. Rev. Lett. 90, 089703 (2003).

11. G. Alvarez, M. Mayr, A. Moreo, and E. Dagotto, Phys. Rev. B71, 014514 (2005).

12. Nanoscale Phase Separation and Colossal Magnetoresistance, E. Dagotto (ed), Springer Verlag, Berlin (2002).

13. E. Dagotto, T. Hotta, and A. Moreo, Phys. Rep. 344, 1 (2001).

14. Y. Tokura and N. Nagaosa, Science 288, 462 (2000).

15. M. Mayr, G. Alvarez, A. Moreo, and E. Dagotto, cond-mat/0503727 (2005).

16. S. Sorella, G.B. Martins, F. Becca, C. Gazza, L. Capriotti, A. Parola, and E. Dagotto, Phys. Rev. Lett. 88, 117002 (2002).

17. S. White and D.J. Scalapino, Phys. Rev. Lett. 80, 1272 (1998).

18. M. Moraghebi, S. Yunoki, and A. Moreo, Phys. Rev. Lett. 88, 187001 (2002).

19. M. Moraghebi, S. Yunoki, and A. Moreo, Phys. Rev. B63, 214513 (2001).

20. W.A. Atkinson, P.J. Hirschfeld, and L. Zhu, Phys. Rev. B68, 054501 (2003).

21. A. Ghosal, C. Kallin, and A.J. Berlinsky, Phys. Rev. B66, 214502 (2002).

22. M. Ichioka and K. Machida, J. Phys. Soc. Jpn. 68, 4020 (1999)

23. S. Zhang, Science 275, 1089 (1997).

24. J.M.T. and. D. Axe1, N. Ichikawa, A.R. Moodenbaugh, Y. Nakamura, and S. Uchida, Phys. Rev. Lett. 78, 338 (1997).
25. D. Poilblanc and T.M. Rice, Phys. Rev. B39, 9749 (1989).

26. J. Zaanen and O. Gunnarsson, Phys. Rev. B40, 7391 (1989).

27. V. Emery and S. Kivelson, Physica 209, 597 (1993).

28. V. Emery and S. Kivelson, Physica 235, 189 (1994).

29. H.-D. Chen, S. Capponi, F. Alet, and S.-C. Zhang, Global Phase Diagram of the High $T_{c}$ Cuprates, cond-mat/0312660 (2003).

30. X. Zhou, T. Yoshida, S.A. Kellar, P.V. Bogdanov, E.D. Lu, A. Lanzara, M. Nakamura, T. Noda, T. Kakeshita, H. Eisaki, S. Uchida, A. Fujimori, Z. Hussain, and Z.-X. Shen, Phys. Rev. Lett. 86, 5578 (2001).

31. M. Mayr, G. Alvarez, C. Sen, and E. Dagotto, Phys. Rev. Lett. 94, 217001 (2005).

32. E. Demler, S. Sachdev, and Y. Zhang, Phys. Rev. Lett. 87, 067202 (2001).

33. Y. Sidis, C. Ulrich, P. Bourges, C. Bernhard, C. Niedermayer, L.P. Regnault, N.H. Andersen, and B. Keimer, Phys. Rev. Lett. 86, 4100 (2001).

34. G. Burns, High-Temperature Superconductivity: An Introduction, Academic Press, Inc., San Diego, CA (1992).

35. H. Eisaki, N. Kaneko, D. Feng, A. Damascelli, P. Mang, K. Shen, Z.-X. Shen, and M. Greven, cond-mat/0312570 (2003).

36. T. Yoshida, X.J. Zhou, T. Sasagawa, W.L. Yang, P.V. Bogdanov, A. Lanzara, Z. Hussain, T. Mizokawa, A. Fujimori, H. Eisaki, Z.-X. Shen, T. Kakeshita, and S. Uchida, Phys. Rev. Lett. 91, 027001 (2003).

37. A. Ino, C. Kim, M. Nakamura, T. Yoshida, T. Mizokawa, Z.-X. Shen, A. Fujimori, T. Kakeshita, H. Eisaki, and S. Uchida, Phys. Rev. B62, 4137 (2000).

38. A. Hossein, D. Broun, D. Sheehy, T. Davis, M. Franz, R. Liang, W. Hardy, and D. Bonn, condmat/0312542 (2003).

39. H. Rho, C.S. Snow, S.L. Cooper, Z. Fisk, A. Comment, and J.-P. Ansermet, Phys. Rev. Lett. 88, 127401 (2002). 